\title{
Stable and Unstable Phases of a Linear Multiblock Copolymer Melt
}

\author{
M. W. Matsen", ${ }^{*}$ and M. Schick \\ Department of Physics, FM-15, University of Washington, Seattle, Washington 98195
}

Received March 15, 1994; Revised Manuscript Received August 16, $1994^{\circ}$

\begin{abstract}
We study the microphases of linear multiblock copolymers formed from monodisperse A- and B-type blocks in the limit in which the number of blocks is large. We find the lamellar, gyroid, hexagonal, and cubic microphases to be stable. Monocontinuous catenoid-lamellar and bicontinuous double-diamond phases are nearly stable in the region between the lamellar and hexagonal phases where the gyroid phase is stable. In the weak-segregation limit, the gyroid phase is not stable, and along the lamellar to hexagonal boundaries a bicontinuous catenoid-lamellar phase is close to stability. In the strong-segregation limit, we can examine only the lamellar phase, and we determine the scaling behavior of its period and interfacial width. We conclude that multiblocks exhibit identical scaling exponents as diblocks.
\end{abstract}

\section{Introduction}

Copolymer melts have received much attention over the past few years due in a large part to their interesting microphase separation. For AB-type copolymers, the simplest of the ordered microphases is the lamellar $(\mathrm{L})$ phase in which $A$ and $B$ monomers separate into A-rich and B-rich lamellae. It is observed to occur when the volume fractions of $A$ and $B$ monomers are comparable. If the volume fraction of one type of monomer becomes sufficiently greater than that of the other, the minority component is observed to form cylinders which pack in a hexagonal arrangement, forming the hexagonal $(\mathrm{H})$ phase. With increasing asymmetry in the volume fractions, it becomes favorable for the minority component to form spheres, which then pack in a body-centered cubic arrangement, the cubic $(C)$ phase.

These ordered phases were the only ones to be observed at first in AB-type copolymer systems. ${ }^{1}$ Later it appeared that in the starblock copolymer system, an ordered, bicontinuous, double-diamond (OBDD) phase also exists. ${ }^{2}$ In this phase, the minority component forms two separate interpenetrating diamond lattices. This phase has since been observed at asymmetries between those of the lamellar and hexagonal phases in several other copolymer systems. . $^{1,3-6}$

A few years ago, a catenoid-lamellar (CL) phase ${ }^{3}$ was observed. This is a lamellar phase in which the lamellae of one component are joined by tubes which perforate those of the other. Typically this phase is monocontinuous with only the minority-component lamellae being perforated, but the observations ${ }^{3}$ in the poly(styrene)/poly(butadiene) (PS/PB) diblock copolymer with a PS volume fraction of $f=0.46$ found it to be bicontinuous with holes in all the lamellae. References 7 and 8 report two distinct hexagonally-structured lamellar phases at $f=0.35$ in the poly(ethylenepropylene)/poly (ethylethylene) (PEP/PEE) diblock, but details of their structures have not been completely determined. The results do suggest that the holes within the lamellae are arranged in a triangular lattice and are staggered from one lamella to the next. Recent observations of the monocontinuous phase in copolymerhomopolymer mixtures ${ }^{4,9}$ have also shown that the holes are triangularly arranged. Moreover, Disko et al. ${ }^{9}$ also

\footnotetext{
* To whom correspondence should be addressed.

t Present address: Department of Chemical Engineering and Materials Science, University of Minnesota, Minneapolis, MN 55455.

- Abstract published in Advance ACS Abstracts, October 15, 1994.
}

observed that the holes between adjacent layers of the minority component are staggered. It is still not known if the stacking of the holes is of the form abab... or abcabc.... We shall label phases which have these stackings as $\mathrm{CL}_{\mathrm{ab}}$ and $\mathrm{CL}_{\mathrm{abc}}$, respectively. In initial theoretical work on such phases, ${ }^{10-12}$ it was assumed that the holes within the minority lamellae were arranged hexagonally and that they were aligned between successive lamellae. We denote this particular version of the phase by $\mathrm{CL}_{\mathrm{a}}$. Fredrickson ${ }^{10}$ found that a monocontinuous $\mathrm{CL}_{\mathrm{a}}$ phase is unstable for the diblock system in the strong-segregation limit, while Olvera de la Cruz et al..$^{11}$ predicted a stable bicontinuous $\mathrm{CL}_{\mathrm{a}}$ phase in the weak-segregation limit.

Most recently, another phase has been observed for weak to intermediate segregation in a poly(styrene)/poly(isoprene) (PS/PI) diblock system with a PS volume fraction of $f=0.33 .{ }^{13}$ It has also been observed in a mixture of two different diblocks, both of poly(styrene)/poly(2vinylpyridine) (PS/PVP), one with PS fraction $f=0.35$ and the other with $f=0.40 .1^{14}$ This gyroid $(G)$ phase is similar to the OBDD phase in that the minority component forms two separate interweaving lattices. However, in the latter, the two lattices are 4-fold coordinated, are identical to one another, and have the symmetry $F d 3 m$. The full OBDD structure has the symmetry $P n 3 m$. In the gyroid phase, the two lattices are 3-fold coordinated, are not identical but are mirror images of one another, and have the symmetry $I 4_{1} 32$. The full gyroid structure, consisting of a right- and a left-handed lattice, belongs to the space group $\operatorname{Ia} 3 d .^{15}$

Experimental methods have been developed which produce linear, multiblock, copolymers which have high structural regularity. ${ }^{16,17}$ The multiblock copolymers produced by Kajiyama et al. ${ }^{16}$ have been shown to produce well-defined microphase separation, but, to our knowledge, the phase diagram has not been explored. Spontak et al. ${ }^{5}$ have examined a selection of melts containing multiblocks with homopolymer, but these experiments have also not produced a phase diagram. Theoretical work on the microphase separation of these systems has begun. Benoit and Hadziioannou ${ }^{18}$ examined the disordered phase and calculated the spinodal line for microphase separation. The lamellar phase has been examined by Kavassalis and Whitmore ${ }^{19}$ in the weak-segregation limit and by Zielinski and Spontak ${ }^{20}$ in the strong-segregation limit.

We expect that systems of $A B$ diblocks and those of linear multiblocks comprised of repeating $A B$ diblocks will exhibit the same ordered phases. The reason is that 
the entropy gained by cutting the multiblock copolymer in the middle of each $\mathrm{A}$ and $\mathrm{B}$ block so as to produce independent diblocks is thought to be small. ${ }^{21-23}$ Consequently, the free energy is largely unaffected, and the phase diagram remains almost unchanged. This argument would seem to be particularly valid in the strongsegregation limit. Therefore, we expect the linear multiblocks to exhibit the usual lamellar, hexagonal, and cubic microphases. They might also exhibit one or more of the double-diamond, gyroid, or various catenoid-lamellar phases.

The above argument also implies that in the strongsegregation limit, the period of an ordered phase, $D$, and the width of the interfacial region between $A$ - and $\mathrm{B}$-monomer-rich regions, $w$, will scale in the same way as in the diblock system. ${ }^{22}$ Therefore, we expect the period of an ordered phase to scale as $D \sim \chi^{1 / 6} N^{2 / 3}$ when it is strongly segregated. . $^{1,621,24-28}$ Here $\chi$ is the usual FloryHuggins parameter and $N$ is the degree of polymerization of the $A B$ repeat unit. Similarly, the interfacial width should scale as $w \sim \chi^{-1 / 2} .{ }^{21,24-26,29,30}$ In contrast to this assumed scaling, calculations in refs 20 and 31 suggest that the exponents will change significantly as the number of repeat units in the multiblock increases.

In this paper, we focus on the linear multiblock which we investigate using a self-consistent, mean-field theory. The results presented in refs 18 and 19 indicate that once the number of $A B$ repeat groups in a linear multiblock copolymer becomes of order 10 , the phase behavior saturates to that of the infinite multiblock. Therefore, we consider this limit and examine the stability of the disordered, lamellar, hexagonal, cubic, double-diamond, gyroid, and various catenoid-lamellar phases. From this, we construct a phase diagram. We explore the phase diagram for different Flory-Huggins parameters, degrees of polymerization, $\mathrm{A}$ and $\mathrm{B}$ monomer volume fractions, and ratios of the $\mathrm{A}$ and $\mathrm{B}$ monomer Kuhn lengths. We find, in addition to lamellar, hexagonal, and cubic microphases, that the gyroid phase is also stable. It occurs between the lamellar and hexagonal phases but does not extend to the weak-segregation limit. There we find a bicontinuous catenoid-lamellar $\mathrm{CL}_{\mathrm{abc}}$ phase to be nearly stable. In the region where we find the gyroid phase to be stable, its free energy is only slightly less than that of the monocontinuous $\mathrm{CL}_{\mathrm{ab}}$ and $\mathrm{CL}_{\mathrm{abc}}$ phases, which, in turn, are only slightly lower than that of the OBDD phase. Due to computational limitations, we cannot perform our calculations in the strong-segregation limit and therefore cannot say whether or not the gyroid phase is stable in this limit. While unequal Kuhn lengths cause phase boundaries to shift, we do not find that they stabilize otherwise unstable phases. Finally, we compare the scaling behavior of the wavelength $D$ and interfacial width $w$ of the lamellar phase occurring in the diblock and multiblock systems. We demonstrate that both systems exhibit identical scaling exponents in the strong-segregation limit.

\section{Theory}

We will consider a linear, multiblock copolymer formed from monodisperse A and B blocks consisting of $f N$ and $(1-f) N$ monomers, respectively. Because we take the limit in which the number of blocks is large, the type of block with which the copolymer terminates will be irrelevant. The theory is simpler if we assume both end blocks are of the same kind so that the copolymer is symmetric. Therefore, we consider copolymers with ( $M$ +1 ) A blocks and $M$ B blocks. The total number of monomers in this multiblock copolymer is $(M+f) N$, and the volume it occupies is $(M+f) N / \rho_{0}$, where $\rho_{0}$ is the density of monomers. In the end, we take the limit $M \rightarrow$ $\infty$, and thus a single copolymer fills a macroscopic volume. For this reason, we consider a system containing only one copolymer.

We assume each monomer occupies a fixed volume and, hence, treat the melt as incompressible. There is no loss in generality in assuming that both $\mathrm{A}$ and $\mathrm{B}$ monomers occupy equal volumes. In fact, one may define each type of monomer in a way that it occupies any desired volume, which we take to be $1 / \rho_{0}$. Once the volume is selected, then the statistical Kuhn length is fixed, ${ }^{24,27}$ and in general the $\mathrm{A}$ and $\mathrm{B}$ monomers will have distinct Kuhn lengths, $a_{\mathrm{A}}$ and $a_{\mathrm{B}}$, respectively.

We parametrize the polymer with a variable $s$ such that $s$ starts at 0 at one end of the polymer and increases continuously to $M+f$ at the other end. This parametrization is chosen such that the volume of the polymer between any two points $s_{1}$ and $s_{2}$ is $N\left|s_{1}-s_{2}\right| / \rho_{0}$. Thus, the $\mathrm{A}$ and $\mathrm{B}$ blocks have lengths $f$ and $1-f$, respectively.

Having specified the system, we turn to a brief derivation of the self-consistent-field theory which describes it. In doing so, we follow Hong and Noolandi. ${ }^{32}$ The partition function of the system is written

$$
\begin{aligned}
Z=\int D \mathbf{r}_{\mathrm{p}} P\left[\mathbf{r}_{\mathrm{p}} ; M+f\right] \delta\left[1-\hat{\phi}_{\mathrm{A}}-\hat{\phi}_{\mathrm{B}}\right] \times \\
\exp \left\{-\frac{\rho_{0}}{N} \int \mathrm{d} \mathbf{r} \chi N \hat{\phi}_{\mathrm{A}}(\mathbf{r}) \hat{\phi}_{\mathrm{B}}(\mathbf{r})\right\}
\end{aligned}
$$

where the functional integral is over all space curves, $\mathbf{r}_{\mathrm{p}}(s)$, which can represent the configuration of the polymer. The probability density functional for a given curve is of the form

$P\left[\mathbf{r}_{\mathrm{p}} ; s\right] \propto \exp \left\{-\frac{3}{2 N} \int_{0}^{s} \mathrm{~d} t\left[\frac{\gamma(t)}{a_{\mathrm{A}}{ }^{2}}+\frac{1-\gamma(t)}{a_{\mathrm{B}}{ }^{2}}\right]\left|\frac{\mathrm{d}}{\mathrm{d} t} \mathbf{r}_{\mathbf{p}}(t)\right|^{2}\right\}$

where $\gamma(s)=1$ when $s$ corresponds to an A-monomer region of the copolymer and is 0 for a B-monomer region. The Flory-Huggins parameter, $\chi$, measures the incompatibility between $\mathrm{A}$ and $\mathrm{B}$ monomers. ${ }^{37}$ The dimensionless $\mathrm{A}$ - and $\mathrm{B}$-monomer-density operators in eq 1 are given by

$$
\begin{gathered}
\hat{\phi}_{\mathrm{A}}(\mathbf{r})=\frac{N}{\rho_{0}} \int_{0}^{M+i} \mathrm{~d} s \gamma(s) \delta\left(\mathbf{r}-\mathbf{r}_{\mathrm{p}}(s)\right) \\
\hat{\phi}_{\mathrm{B}}(\mathbf{r})=\frac{N}{\rho_{0}} \int_{0}^{M+f} \mathrm{~d} s(1-\gamma(\mathbf{s})) \delta\left(\mathbf{r}-\mathbf{r}_{\mathrm{p}}(s)\right)
\end{gathered}
$$

These densities depend upon the configuration of the polymer, $\mathbf{r}_{\mathrm{p}}(s)$, and it is the product of density operators in eq 1 , representing the interaction of one part of the polymer with another, which makes the evaluation of the partition function so difficult. To make it more tractable, one inserts functional integrals over delta functionals, 1 $=\int D \Phi_{\mathrm{A}} D \Phi_{\mathrm{B}} \delta\left[\Phi_{\mathrm{A}}-\hat{\phi}_{\mathrm{A}}\right] \delta\left[\Phi_{\mathrm{B}}-\hat{\phi}_{\mathrm{B}}\right]$, which permits the replacement of the product of operators, $\hat{\phi}_{A} \hat{\phi}_{B}$, in eq 1 by the product of functions, $\Phi_{A} \Phi_{B}$, as well as the replacement of operators by functions in $\delta\left[1-\hat{\phi}_{A}-\hat{\phi}_{B}\right]$. One then inserts an integral representation for the delta functionals of the 
form

$$
\begin{aligned}
& \delta\left[\Phi_{\mathrm{A}}-\hat{\phi}_{\mathrm{A}}\right]=\mathcal{N} \int \mathcal{D} W_{\mathrm{A}} \times \\
& \quad \exp \left\{\frac{\rho_{0}}{N} \int \mathrm{d} \mathbf{r} W_{\mathrm{A}}(\mathbf{r})\left[\Phi_{\mathrm{A}}(\mathbf{r})-\hat{\phi}_{\mathrm{A}}(\mathbf{r})\right]\right\} \\
& \delta\left[1-\Phi_{\mathrm{A}}-\Phi_{\mathrm{B}}\right]= \mathcal{N} \int \mathcal{D} \Xi \times \\
& \exp \left\{\frac{\rho_{0}}{N} \int \mathrm{d} \mathbf{r} \Xi(\mathbf{r})\left[1-\Phi_{\mathrm{A}}(\mathbf{r})-\Phi_{\mathrm{B}}(\mathbf{r})\right]\right\}
\end{aligned}
$$

where $\mathcal{N}$ is a normalization constant and the limits of integration are $\pm i \infty$. These insertions bring the partition function into the form

$$
\begin{array}{rl}
Z=\mathcal{N}^{3} \int \mathcal{D} \Phi_{\mathrm{A}} D W_{\mathrm{A}} & D \Phi_{\mathrm{B}} \mathscr{D} W_{\mathrm{B}} \mathscr{D} \Xi \times \\
& \exp \left\{-F\left[\Phi_{\mathrm{A}}, W_{\mathrm{A}}, \Phi_{\mathrm{B}}, W_{\mathrm{B}}, \Xi\right] / k_{\mathrm{B}} T\right\}
\end{array}
$$

with

$$
\begin{aligned}
& F\left[\Phi_{\mathrm{A}}, W_{\mathrm{A}}, \Phi_{\mathrm{B}}, W_{\mathrm{B}}, \Xi\right] / k_{\mathrm{B}} T \equiv- \\
& \frac{\rho_{0}}{N} \int \mathrm{d} \mathbf{Q}\left[W_{\mathrm{A}}, W_{\mathrm{B}}\right]+ \\
& \chi \chi N \Phi_{\mathrm{A}}(\mathbf{r}) \Phi_{\mathrm{B}}(\mathbf{r})-W_{\mathrm{A}}(\mathbf{r}) \Phi_{\mathrm{A}}(\mathbf{r})-W_{\mathrm{B}}(\mathbf{r}) \Phi_{\mathrm{B}}(\mathbf{r})- \\
& \left.\Xi(\mathbf{r})\left(1-\Phi_{\mathrm{A}}(\mathbf{r})-\Phi_{\mathrm{B}}(\mathbf{r})\right)\right]
\end{aligned}
$$

and

$$
\begin{aligned}
& Q\left[W_{\mathrm{A}}, W_{\mathrm{B}}\right] \equiv \int D \mathbf{r}_{\mathrm{p}} P\left[\mathbf{r}_{\mathrm{p}} ; M+f\right] \times \\
& \exp \left\{-\frac{\rho_{0}}{N} \int \mathrm{d} \mathbf{r}\left[W_{\mathrm{A}}(\mathbf{r}) \hat{\phi}_{\mathrm{A}}(\mathbf{r})+W_{\mathrm{B}}(\mathbf{r}) \hat{\phi}_{\mathrm{B}}(\mathbf{r})\right]\right\}(9) \\
& \quad=\int D \mathbf{r}_{\mathrm{p}} P\left[\mathbf{r}_{\mathrm{p}} ; M+f\right] \times \\
& \exp \left\{-\int_{0}^{M+f} \mathrm{~d} s\left[\gamma(s) W_{\mathrm{A}}\left(\mathbf{r}_{\mathrm{p}}(s)\right)+(1-\gamma(s)) W_{\mathrm{B}}\left(\mathbf{r}_{\mathrm{p}}(s)\right)\right]\right\}
\end{aligned}
$$

This last quantity, $Q\left[W_{\mathrm{A}}, W_{\mathrm{B}}\right]$, is simply the partition function of a noninteracting polymer in external fields, $W_{\mathrm{A}}(\mathbf{r})$ and $W_{\mathrm{B}}(\mathbf{r})$. The above transformation is exact.

What has been accomplished is to replace the quadratic occurrence of operators in eq 1 by the linear occurrence in $Q$ which, as noted below, can be calculated exactly. Nonetheless, the functional integrals in eq 7 cannot be carried out. The self-consistent-field approximation is to evaluate the partition function of eq 7 by the method of steepest descents and thereby approximate the free energy, $-k_{\mathrm{B}} T \ln Z$, by the extremum of $F\left[\Phi_{\mathrm{A}}, W_{\mathrm{A}}, \Phi_{\mathrm{B}}, W_{\mathrm{B}}, \Xi\right]$. We denote the extremum $F\left[\phi_{\mathrm{A}}, w_{\mathrm{A}}, \phi_{\mathrm{B}}, w_{\mathrm{B}}, \xi\right]$. The functions at which the extremum is attained are found from eq 8 . Variation with respect to $\Phi_{A}(\mathbf{r})$ and to $\Phi_{B}(\mathbf{r})$ yields

$$
\begin{aligned}
& w_{\mathrm{A}}(\mathbf{r})=\chi N \phi_{\mathrm{B}}(\mathbf{r})+\xi(\mathbf{r}) \\
& w_{\mathrm{B}}(\mathbf{r})=\chi N \phi_{\mathrm{A}}(\mathbf{r})+\xi(\mathbf{r})
\end{aligned}
$$

while variation with respect to $\Xi(\mathbf{r}), W_{\mathrm{A}}(\mathbf{r})$, and $W_{\mathrm{B}}(\mathbf{r})$ gives

$$
\begin{gathered}
\phi_{\mathrm{A}}(\mathbf{r})+\phi_{\mathrm{B}}(\mathbf{r})=1 \\
\phi_{\mathrm{A}}=-\frac{N}{\rho_{0}} \frac{1}{Q} \frac{D Q Q}{D w_{\mathrm{A}}} \\
\phi_{\mathrm{B}}=-\frac{N}{\rho_{0}} \frac{1}{\mathscr{Q}} \frac{D \mathcal{Q}}{D w_{\mathrm{B}}}
\end{gathered}
$$

The last two equations identify $\phi_{\mathrm{A}}(\mathbf{r})$ and $\phi_{\mathrm{B}}(\mathbf{r})$ as the average densities of $A$ and $B$ monomers at $r$ as calculated in the ensemble of the noninteracting polymer subject to external fields which are calculated self-consistently from eqs 11 and 12. Thus, the saddle-point approximation has reduced the problem of an interacting polymer to one of a noninteracting polymer in fixed external fields. Once the partition function of the latter problem, $Q\left[w_{\mathrm{A}}, w_{\mathrm{B}}\right]$, is known, the set of equations 11-15 can be solved.

It is at this point that additional approximations are often made, such as expanding $\ln Q$ in cumulants and keeping only the first few terms in the expansion. ${ }^{11,12,33}$ Such additional approximations may be convenient at times but are unnecessary because the partition function $Q$ can be obtained exactly. One writes the partition function in terms of the end-segment distribution function $q(\mathbf{r}, s)$,

$$
Q=\int \mathrm{d} \mathbf{r} q(\mathbf{r}, M+f)
$$

where

$$
\begin{aligned}
& q(\mathbf{r}, s)=\int \mathcal{D} \mathbf{r}_{\mathrm{p}} P\left[\mathbf{r}_{\mathrm{p}} ; s\right] \delta\left(\mathbf{r}-\mathbf{r}_{\mathrm{p}}(s)\right) \times \\
& \quad \exp \left\{-\int_{0}^{s} \mathrm{~d} t\left[\gamma(t) w_{\mathrm{A}}\left(\mathbf{r}_{\mathrm{p}}(t)\right)+(1-\gamma(t)) w_{\mathrm{B}}\left(\mathbf{r}_{\mathrm{p}}(t)\right)\right]\right\}
\end{aligned}
$$

It satisfies the modified diffusion equation ${ }^{34}$

$$
\frac{\partial}{\partial s} q(\mathbf{r}, s)= \begin{cases}\frac{1}{6} N a_{A}^{2} \nabla^{2} q(\mathbf{r}, s)-w_{A}(\mathbf{r}) q(\mathbf{r}, s), & \text { if } \gamma(s)=1 \\ \frac{1}{6} N a_{B}^{2} \nabla^{2} q(\mathbf{r}, s)-w_{B}(\mathbf{r}) q(\mathbf{r}, s), & \text { otherwise }\end{cases}
$$

and, from its definition eq 17 , the initial condition, $q(\mathbf{r}, 0)$ $=1$, for all $\mathbf{r}$. In terms of the end-segment distribution function, the A-monomer density (eq 14) can be written

$$
\phi_{\mathrm{A}}(\mathbf{r})=\frac{N}{\rho_{0} Q} \int_{0}^{M+f} \mathrm{~d} s \gamma(s) q(\mathbf{r}, s) q(\mathbf{r}, M+f-s)
$$

where the fact that the polymer is symmetric has been used to convert an integral along the chain from $M+f$ to $s$ to one from 0 to $M+f-s$. The expression for $\phi_{\mathrm{B}}(\mathbf{r})$ is similar.

Once a solution of the self-consistent equations is found, the free energy of that solution is $F\left[\phi_{\mathrm{A}}, w_{\mathrm{A}}, \phi_{\mathrm{B}}, w_{\mathrm{B}}, \xi\right]$ of eq 8. Rather than attempting to solve the above selfconsistent equations in real space, we expand all functions of position in a convenient set of orthonormal basis functions $f_{i}(\mathbf{r}), i=1,2,3, \ldots$ :

$$
g(\mathbf{r})=\sum_{i} g_{i} f_{i}(\mathbf{r})
$$

with

$$
\frac{1}{V_{\text {cell }}} \int_{\text {cell }} \mathrm{d} \mathbf{r} f_{i}(\mathbf{r}) f_{j}(\mathbf{r})=\delta_{i j}
$$


We take the basis functions to be eigenfunctions of the Laplacian operator:

$$
\nabla^{2} f_{i}(\mathbf{r})=-\frac{\lambda_{i}}{D^{2}} f_{i}(\mathbf{r})
$$

The length scale, $D$, and the choice of $V_{\text {cell }}$ are discussed below. We order the functions such that $\lambda_{i}$ is a nondecreasing series. The first basis function is then $f_{1}(\mathbf{r})=1$ with an eigenvalue $\lambda_{1}=0 . .^{35}$

In this basis, the diffusion equation (18) for $q(\mathbf{r}, s)$ becomes

$$
\frac{\mathrm{d} q_{i}(s)}{\mathrm{d} s}= \begin{cases}\sum_{j} A_{i j} q_{j}(s), & \text { if } \gamma(s)=1 \\ \sum_{j} B_{i j} q_{j}(s), & \text { otherwise }\end{cases}
$$

where the matrix, $A$, is given by

$$
A_{i j}=-\frac{N a_{\mathrm{A}}{ }^{2} \lambda_{i}}{6 D^{2}} \delta_{i j}-\sum_{k} w_{\mathrm{A}, k} \Gamma_{i j k}
$$

with

$$
\Gamma_{i j k}=\frac{1}{V_{\text {ceil }}} \int_{\text {cell }} \mathrm{d} \mathbf{r} f_{i}(\mathbf{r}) f_{j}(\mathbf{r}) f_{k}(\mathbf{r})
$$

The matrix, $B$, is given by a similar expression. The initial condition is $q_{i}(0)=\delta_{i 1}$.

Given the amplitudes $w_{\mathrm{A}, i}$ and $w_{\mathrm{B}, i}$ of the fields, we can easily solve the diffusion equation for $q_{i}(s)$ (eq 23) in a basis in which the symmetric matrix $A$ is diagonal, if $\gamma(s)$ $=1$, or $B$ is diagonal, if $\gamma(s)=0$. Orthogonal transformations accomplish this. After transformation back to the original basis, the solution can be put in the form

$$
q_{i}\left(s+s^{\prime}\right)=\sum_{j} T_{\mathrm{A}, i j}\left(s^{\prime}\right) q_{j}(s)
$$

if there are only A monomers between $s$ and $s+s^{\prime}$, and

$$
q_{i}\left(s+s^{\prime}\right)=\sum_{j} T_{\mathrm{B}, i j}\left(s^{\prime}\right) q_{j}(s)
$$

if there are only $B$ monomers between them. Given the above initial condition for $q_{i}(0)$, these transfer matrices, $T_{\mathrm{A}}(s)$ and $T_{\mathrm{B}}(s)$, produce the amplitudes $q_{i}(s)$ elsewhere along the chain. At the end of a complete AB block, $s$ takes on an integer value, $m$, and the combined transfer matrix,

$$
T_{i j}=\sum_{k} T_{\mathrm{B}, i k}(1-f) T_{\mathrm{A}, k j}(f)
$$

moves $q_{j}(m)$ through such an AB block to $q_{i}(m+1)$. In calculating $q_{i}(s)$ for $m<s<m+1$, the transfer matrix operates $m$ times on $q_{j}(0)$. In the limit where the chain becomes infinite, we can ignore end effects and always assume $m \gg 1$. Therefore, the eigenvector of $T$ with the largest eigenvalue dominates all calculated quantities. For example, the dependence of the configuration integral on the number of blocks $M$ is $Q \sim \epsilon^{M}$, where we have denoted the largest eigenvalue by $\epsilon$. We shall denote the corresponding right and left eigenvectors, $\psi_{\mathrm{R}, i}$ and $\psi_{\mathrm{L}, i}$, respectively. The distinction is necessary because $T$ is not a symmetric matrix, even though $T_{\mathrm{A}}$ and $T_{\mathrm{B}}$ are.

Now that the amplitudes of the end-segment distribution functions are known, the amplitudes of the densities are obtained from eq 19

$$
\phi_{\mathrm{A}, i}=\frac{1}{C_{\mathrm{A}}} \int_{0}^{f} \mathrm{~d} s \sum_{j k l m} \psi_{\mathrm{R}, j} \psi_{\mathrm{R}, k} T_{\mathrm{A}, j l}(s) T_{\mathrm{A}, k m}(f-s) \Gamma_{l m i}
$$

where

$$
C_{\mathrm{A}}=\sum_{j k} \psi_{\mathrm{R}, j} \psi_{\mathrm{R}, k} T_{\mathrm{A}, j k}(f)
$$

and

$\phi_{\mathrm{B}, i}=\frac{1}{C_{\mathrm{B}}} \int_{0}^{1-f} \mathrm{~d} s \sum_{j k l m} \psi_{\mathrm{L}, j} \psi_{\mathrm{L}, k} T_{\mathrm{B}, j l}(s) T_{\mathrm{B}, k m}(1-f-s) \Gamma_{l m i}$

with

$$
C_{\mathrm{B}}=\sum_{j k} \psi_{\mathrm{L}, j} \psi_{\mathrm{L}, k} T_{\mathrm{B}, j k}(1-f)
$$

We must now adjust the assumed amplitudes $w_{\mathrm{A}, i}$ and $w_{\mathrm{B}, i}$ of the fields so that the densities calculated from them above satisfy the incompressibility condition, eq 13 ,

$$
\phi_{\mathrm{A}, i}+\phi_{\mathrm{B}, i}=0, \quad i=2,3, \ldots
$$

and, in addition, are related to the monomer-density amplitudes via eqs 11 and 12:

$$
\omega_{\mathrm{A}, i}-\omega_{\mathrm{B}, i}+\chi N\left(\phi_{\mathrm{A}, i}-\phi_{\mathrm{B}, i}\right)=0, \quad i=2,3, \ldots
$$

Without loss of generality, we can set the average value of $\xi(\mathbf{r})$, which is $\xi_{1}$, to zero so that $\omega_{\mathrm{A}, 1}=\chi N \phi_{\mathrm{B}, 1}=\chi N(1$ - f) and $\omega_{\mathrm{B}, 1}=\chi N \phi_{\mathrm{A}, 1}=\chi N f$. This completes the cycle of self-consistent equations.

With our choice of $\xi(\mathbf{r})$, the free energy of eq 8 can be written

$$
F / M k_{\mathrm{B}} T=-\ln (\epsilon)-\chi N \sum_{i} \phi_{\mathrm{A}, i} \phi_{\mathrm{B}, i}
$$

For the disordered phase, $\epsilon=\exp \left(-\omega_{\mathrm{A}, 1} f-\omega_{\mathrm{B}, 1}(1-f)\right)$, and thus its free energy is $F / M k_{\mathrm{B}} T=\chi N f(1-f)$. For a periodic, ordered phase of a certain symmetry, only eigenfunctions of the Laplacian with that symmetry ${ }^{35}$ are included in the sequence of functions, $f_{i}(\mathbf{r})$. They are normalized within a unit cell of the phase, $V_{\text {cell. }}$. The free energy still has to be minimized with respect to the wavelength, $D$, of the phase. For catenoid-lamellar phases, there are two such wavelengths: one of the lamellae and the other of the triangular arrangement of holes within each sheet. By comparing the free energies for different phases, we determine the phase diagram.

As a matter of practicality, only a finite number of basis functions are included in the expansion of spatiallydependent quantities. We have used a sufficient number of basis functions to achieve an accuracy of, at least, a part in $10^{4}$ in the free energy. The number of functions needed to achieve this depends on the phase examined and the value of $\chi N$. We have employed up to 60 of them.

\section{Results}

In Figures $1-3$ we show the phase diagrams we have calculated for Kuhn lengths with the ratios of $a_{\mathrm{A}} / a_{\mathrm{B}}=1$, 1.5 , and 2 , respectively. These are reasonable values. ${ }^{36}$ One can see that unequal Kuhn lengths produce the type of asymmetry in the phase diagram that is seen experimentally. $., 8,37$ The most notable aspect of these diagrams is that, in addition to the usual lamellar, hexagonal, and 


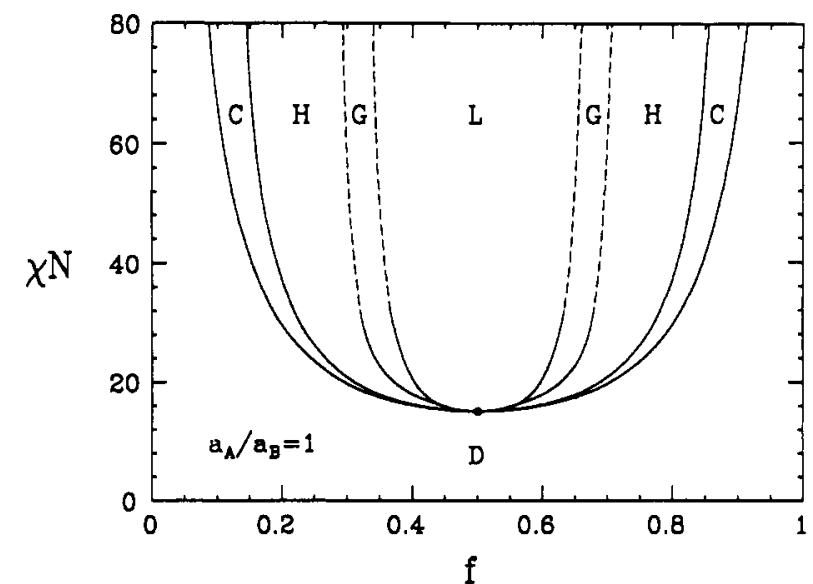

Figure 1. Phase diagram for linear multiblock copolymers with equal Kuhn lengths. The regions of stability for the disordered, lamellar, gyroid, hexagonal, and cubic phases are denoted D, L, $\mathrm{G}, \mathrm{H}$, and $\mathrm{C}$, respectively. All transitions are first order except for the critical point which is marked by a dot. Dashed lines denote extrapolated phase boundaries.

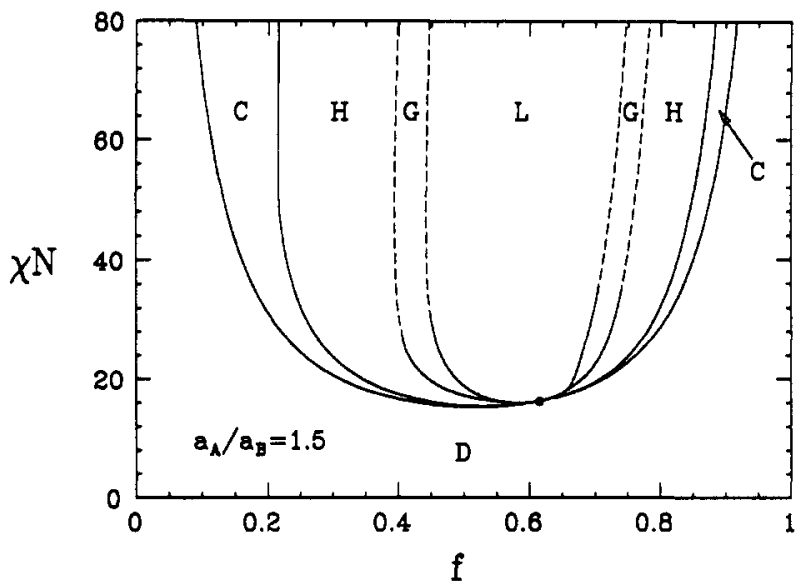

Figure 2. Phase diagram for copolymers with a ratio of Kuhn lengths of $2: 3$.

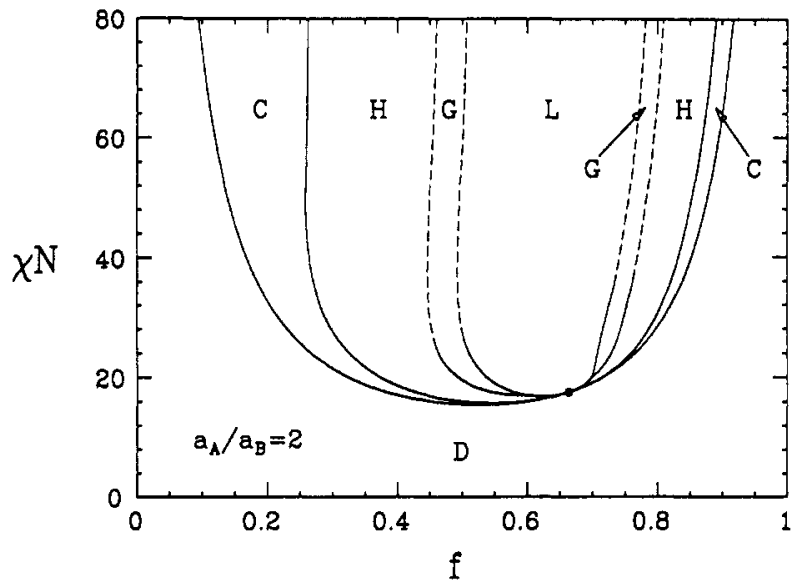

Figure 3. Phase diagram for copolymers with a ratio of Kuhn lengths of $1: 2$.

cubic phases, there exists a stable gyroid phase which, when it exists, is found between the lamellar and hexagonal phases. Unlike the other phases which all extend to a critical point which we indicate with a dot, the $G$ phases terminate at triple points, leaving short lines of lamellar to hexagonal transitions in the weak-segregation limit. We could not calculate the phase boundaries of the $G$ phase in the strong-segregation limit because of the large number of basis functions required. We have made the assumption that, as $\chi N \rightarrow \infty$, this phase attains a nearly uniform width along the metastable $\mathrm{L}-\mathrm{H}$ transition which we could
Table 1. Free Energy Excess with Respect to the Stable

Gyroid Phase, $\Delta F$, and Characteristic Length, $D$, for Various Phases at $\chi N=20$ and $f=0.3933$ with Both Kuhn Lengths Equal to $a^{*}$

\begin{tabular}{llcl}
\hline phase & $\Delta F / M k_{\mathrm{B}} T$ & $D / a N^{1 / 2}$ & $C / D$ \\
\hline $\mathrm{G}$ & 0.0 & 2.659 & \\
$\mathrm{MCL}_{\mathrm{ab}}$ & 0.00302 & 1.079 & 1.308 \\
$\mathrm{MCL}_{\mathrm{abc}}$ & 0.00321 & 1.080 & 1.311 \\
$\mathrm{~L}$ & 0.00470 & 1.083 & \\
$\mathrm{H}$ & 0.00470 & 1.236 & \\
$\mathrm{OBDD}_{\mathrm{BCL}_{\mathrm{abc}}}$ & 0.01385 & 1.611 & \\
$\mathrm{BCL}_{\mathrm{ab}}$ & 0.01823 & 1.080 & 1.224 \\
$\mathrm{BCL}_{\mathrm{a}}$ & 0.02386 & 1.142 & 1.140 \\
$\mathrm{C}$ & 0.02685 & 1.098 & 1.143 \\
$\mathrm{D}$ & 0.02715 & 1.513 & \\
\hline
\end{tabular}

${ }^{a}$ For the $\mathrm{L}$ and $\mathrm{CL}$ phases, $D$ is the lamellar spacing, and for the $H$ phase, it is the spacing of the cylinders. For the $\mathrm{C}, \mathrm{G}$, and OBDD phases, it is the size of their cubic unit cells. The ratio of the inplane, $C$, to out-of-plane, $D$, lattice parameters is given for $C L$ phases.

calculate. With this assumption, we have extrapolated the phase boundaries up to $\chi N=80$ using dashed lines.

In addition to the gyroid phase, we find a number of other phases which compete for stability between the lamellar and hexagonal phases. In Table 1, we compare their relative stabilities at $\chi N=20$ on the metastable $\mathrm{L}-\mathrm{H}$ transition with the ratio of Kuhn lengths equal to unity. For this case, the A monomer fraction is $f=0.3933$. We have indicated whether the catenoid-lamellar solutions are monocontinuous or bicontinuous by the letter $\mathrm{M}$ or $\mathrm{B}$ before the designation CL. In the second column of the table, we give the difference between the free energy of each phase and that of the stable gyroid phase. The wavelength in units of the Kuhn length, $a$, is given in the third column, and, for the CL phases, the ratio of the lattice parameter in the plane of the lamellae to that perpendicular to them is given in column four.

In all the regions where the $G$ phase is stable, the two monocontinuous catenoid-lamellar phases, $\mathbf{M C L}_{\mathrm{ab}}$ and $\mathrm{MCL}_{\mathrm{abc}}$, are nearly stable. Both these have holes in the minority-component lamellae through which the majoritycomponent ones are connected. The holes are arranged hexagonally within the layers and stagger between adjacent perforated layers. The perforated lamellae are stacked in abab... and abcabc... sequences, respectively. No solution was found for a monocontinuous CL phase with aligned holes. The two solutions with staggered holes are nearly degenerate in free energy, but the $\mathrm{MCL}_{\mathrm{ab}}$ is slightly favored. In both phases, the lamellar spacing is nearly the same as that for the metastable $\mathrm{L}$ phase, and the ratio of the hole spacing to the lamellar spacing varies between 1.3 and 1.4, in the region where they are nearly stable. For the larger $\chi N$ values which we could explore on the metastable $\mathrm{L}-\mathrm{H}$ transition, the OBDD phase was also found to be nearly stable, but it was less stable than the above two CL phases and, in fact, less stable than the $\mathrm{L}$ and $\mathrm{H}$ phases.

The bicontinuous CL phases are most favored at weak segregations along the $\mathrm{L}$ to $\mathrm{H}$ phase boundaries close to the critical points. Of these phases, the bicontinuous $\mathrm{CL}_{\mathrm{abc}}$ phase is the most nearly stable. At the triple point, $x N$ $=16.38$ and $f=0.4376$, with $a_{\mathrm{A}}=a_{\mathrm{B}}$, where the $\mathrm{L}, \mathrm{H}$, and $G$ phases coexist, the excess free energy of the $B C L_{a b c}$ phase is $\Delta F / M k_{\mathrm{B}} T=0.00171$ as compared to 0.01032 for the disordered phase. In this phase, the ratio of the hole spacing to the lamellar spacing remains nearly equal to $(3 / 2)^{1 / 2}$, the close-packing ratio.

We now focus on the symmetric multiblock with $f=0.5$ and $a_{\mathrm{A}}=a_{\mathrm{B}} \equiv a$. The profile within its lamellar phase at $\chi N=200$ is shown in Figure 4. The period, $D / a N^{1 / 2}=$ 


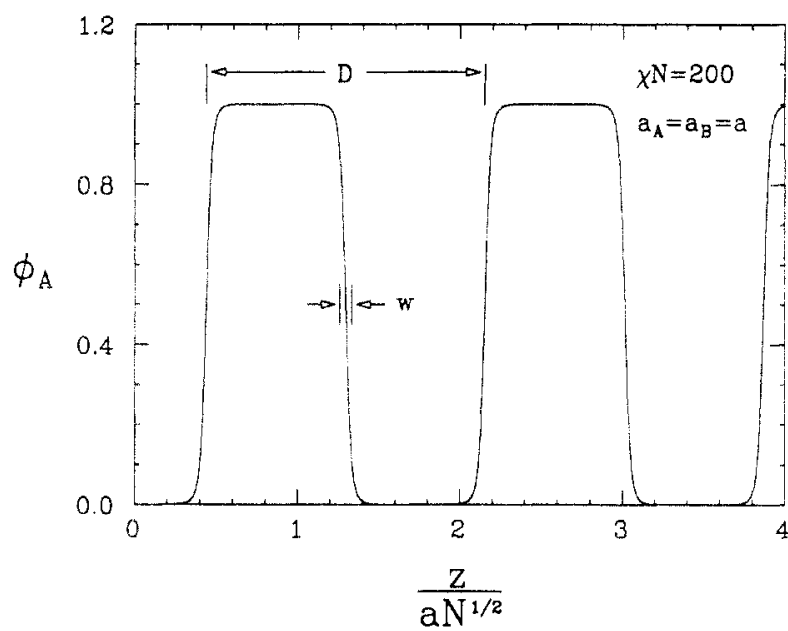

Figure 4. Plot of the A monomer profile in the lamellar phase of a symmetric linear multiblock in the strong-segregation limit. The dimensions of the lamellar spacing, $D$, and the interfacial width, $w$, are explicitly indicated.

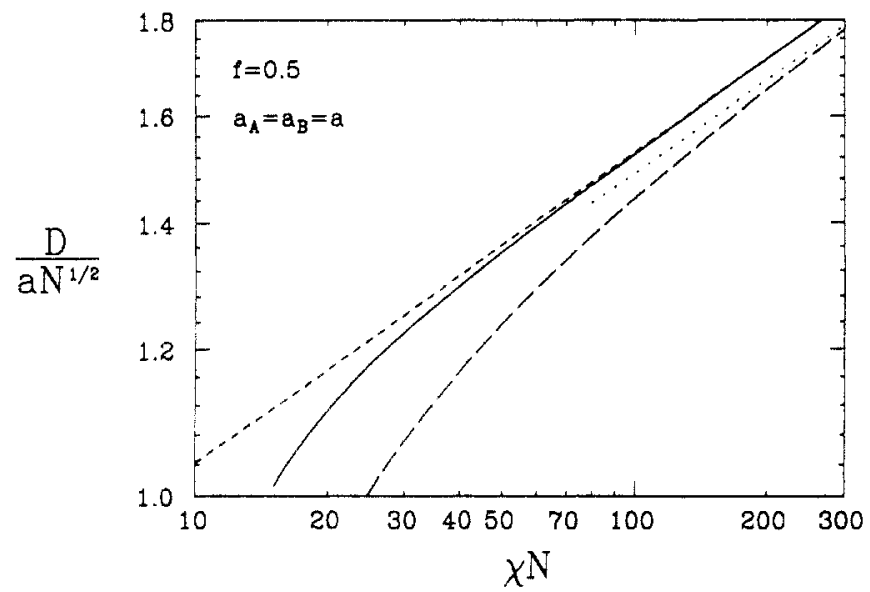

Figure 5. $\log -\log$ plot of the lamellar spacing versus $\chi N$ for symmetric copolymers. The solid line corresponds the infinite linear multiblock, and the short-dashed line is a strong-segregation fit to it. The long-dashed line corresponds to a system of diblock with degree of polymerization $N / 2$.

1.7165 , and width of the interfaces between the lamellae, $w / a N^{1 / 2}=0.07041$, are indicated on the figure. For the interfacial width, we use the definition $24,25,29,38$

$$
w=\left(\mathrm{d} \phi_{\mathrm{A}} / \mathrm{d} z\right)_{z=\mathrm{z}_{0}}^{-1}
$$

where the $z$-direction is taken to be orthogonal to the lamellae and $\phi_{\mathrm{A}}\left(z_{0}\right)=f$. In Figures 5 and 6 , we plot $D$ and $w$ with solid lines using logarithmic scales to demonstrate the power-law behavior in the strong-segregation limit. For comparison, we also plot with long-dashed lines what $D$ and $w$ would be if all the A and B blocks were cut at their midpoints, producing a system of AB diblocks. One can see that while this has little effect on the interfacial width, $w$, it has a sizable effect on $D$. This occurs because cutting the multiblock gives the polymer chain more configurational freedom, resulting in more entropy which reduces the chain stretching, leading to a decrease in the lamellar spacing. This effect is reduced in the strong-segregation limit, but it is never negligible, contrary to what one might have expected.

Fitting the strong-segregation limit of the lamellar spacing to

$$
D / a N^{1 / 2}=c(\chi N)^{b}
$$

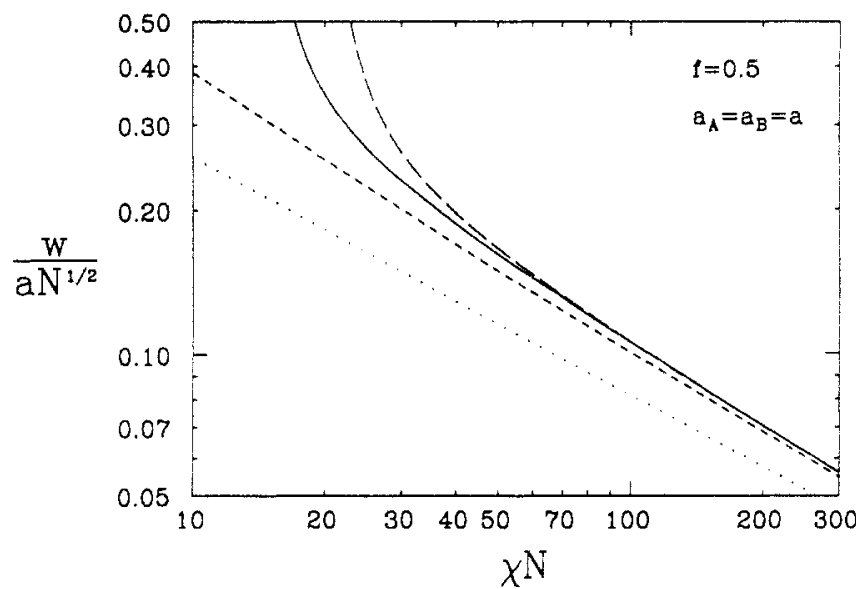

Figure 6. $\log$-log plot of the interfacial width between lamellae for symmetric copolymers. The solid line corresponds to the infinite linear multiblock and the long-dashed line to the diblocks with degree of polymerization $N / 2$. The dotted line is the interfacial width between infinitely long incompatible homopolymers, and the short-dashed line is the approximation eq 38 , derived by Semenov. 30

we find $c=0.710$ and $b=0.167$. This fit is shown with the short-dashed line in Figure 5. This result for $b$ is consistent with $b=1 / 6$. We have calculated the spacing $D$ for the diblock system at values of $\chi N$ beyond the range shown so that the segregation of the lamellar phase becomes comparable to that for the multiblock system within this range and have fitted the result for $D$ to the same functional form. The result yields the same value for the exponent $b$, but a smaller value, $c=0.692$, for the proportionality constant. This value of the constant is consistent with that calculated by Semenov, ${ }^{26,39} c=$ $2 /\left(6 \pi^{4}\right)^{1 / 6}=0.6917$.

Figure 6 demonstrates that the interfacial widths in the multiblock system (solid line) and that the diblock system (long-dashed line) which would be produced by cutting the midpoint of each $A$ and $B$ block become identical in the strong-segregation limit. Shull ${ }^{24}$ has argued that, in this limit, the interfacial width in the microphases of copolymer melts should be the same as those between incompatible homopolymers with infinite degrees of polymerization, $w / a=2 /(6 \chi)^{1 / 2} .38$ This expression is shown with a dotted line in Figure 6. As noted by Shull for the case of diblocks, one must consider a very strongly segregated melt before this becomes a good approximation. Clearly, a better approximation for the strong-segregation limit is desirable. ${ }^{29}$ Semenov ${ }^{30}$ has recently derived one, ${ }^{39}$

$$
\frac{w}{a N^{1 / 2}} \approx \frac{2}{(6 \chi N)^{1 / 2}}\left[1+\frac{4}{\pi}\left(\frac{6}{\pi^{2} \chi N}\right)^{1 / 3}\right]
$$

This approximation, which we show in Figure 6 with a short-dashed line, agrees quite well with our calculation.

\section{Conclusions}

We have calculated the relative stability of several ordered phases of various symmetries for a system consisting of an infinite, linear, multiblock copolymer. Our results are similar to those which we obtained for diblocks, ${ }^{40}$ as would be expected. We found that, in addition to the lamellar, hexagonal, and cubic phases, the gyroid phase is also stable. It exists between the lamellar and hexagonal phases but does not extend all the way to the weaksegregation limit. Although this phase has not yet been observed in the multiblock system, its location in the phase diagram is consistent with observations on diblock systems. ${ }^{13,14}$ 
Also of interest are the phases which are nearly stable between the lamellar and hexagonal ones. We could not explore strong segregations, but the trends we obtained indicate that the OBDD may become stable in this limit. At intermediate segregations, we found that two monocontinuous catenoid-lamellar phases are close to being stable. Both these phases are characterized by a triangular array of tubes which penetrate the minority lamellae and connect the majority ones. In these phases, $\mathrm{CL}_{\mathrm{ab}}$ and $\mathrm{CL}_{\mathrm{abc}}$, the tubes are staggered in abab... and abcabc... sequences, respectively. Although the former, $\mathrm{CL}_{\mathrm{ab}}$, is favored, they have nearly degenerate free energies and their dimensions are very similar. Along the lines of coexistence between the lamellar and hexagonal phases, which exist in the weaksegregation limit, we find a bicontinuous catenoid-lamellar phase to be nearly stable. In this phase every lamellae is perforated by holes, and the stacking of the layers occurs in an abcabc... sequence.

We have also considered the effect of unequal Kuhn lengths of the $\mathrm{A}$ and $\mathrm{B}$ monomers on the phase diagram. We generated them for cases for which the ratio of these lengths is $1: 1,2: 3$, and $1: 2$, values which correspond approximately to PS/PI, PEP/PEE, and PE/PEE copolymer systems, respectively, ${ }^{8,36}$ This changes the locations of the various phases in accord with experiment, making some regions of stability larger and others smaller. For instance, the region of stability of the gyroid phase is enhanced if the ratio of the minority- to majoritycomponent Kuhn lengths is increased.

We have examined both the period $D$ and interfacial width $w$ in the lamellar phase of the multiblock system. In the strong-segregation limit, we find that these quantities scale with the same exponents as for the diblock system; i.e., $D \sim \chi^{1 / 6} N^{2 / 3}$ and $w \sim \chi^{-1 / 2}$. Although we have not examined the scaling for the other morphologies, the scaling exponents for them should be the same as that for the lamellar one.

Acknowledgment. We acknowledge useful conversations with A.-C. Shi, E. L. Thomas, F. S. Bates, S. Milner, and R. A. Sones. We are also grateful to the Department of Physics at Simon Fraser University for use of their computing facilities. This work was supported in part by the National Science Foundation under Grant No. DMR 9220733 and the Natural Science and Engineering Research Council of Canada.

\section{References and Notes}

(1) Hasegawa, H.; Tanaka, H.; Yamasaki, K.; Hashimoto, T. Macromolecules 1987, 20, 1651 .

(2) Alward, D. B.; Kinning, D. J.; Thomas, E. L.; Fetters, L. J. Macromolecules 1986, 19, 215. Thomas, E. L.; Alward, D. B.; Kinning, D. J.; Martin, D. C.; Handlin, D. L., Jr.; Fetters, L. J. Macromolecules 1986, 19, 2197.

(3) Thomas, E. L.; Anderson, D. M.; Henkee, C. S.; Hoffman, D. Nature 1988, 334, 598.

(4) Spontak, R. J.; Smith, S. D.; Ashraf, A. Macromolecules 1993, 26, 956. Hashimoto, T.; Koizumi, S.; Hasegawa, H.; Izumitani,
T.; Hyde, S. T. Macromolecules 1992, 25, 1433.

(5) Spontak, R. J.; Smith, S. D.; Ashraf, A. Macromolecules 1993, 26,5118 .

(6) Hashimoto, T.; Yamasaki, K.; Koizumi, S.; Hasegawa, H. Macromolecules 1993, 26, 2895.

(7) Hamley, I. W.; Koppi, K. A.; Rosedale, J.H.; Bates, F. S.; Almdal, K.; Mortensen, K. Macromolecules 1993, 26, 5959.

(8) Almdal, K.; Koppi, K. A.; Bates, F. S.; Mortensen, K. Macromolecules 1992, 25, 1743.

(9) Disko, M. M.; Liang, K. S.; Behal, S. K.; Roe, R. J.; Jeon, K. J. Macromolecules 1993, 26, 2983.

(10) Fredrickson, G. H. Macromolecules 1991, 24, 3456.

(11) Olvera de la Cruz, M.; Mayes, A. M.;Swift, B. W. Macromolecules $1992,25,944$.

(12) Olvera de la Cruz, M. Phys. Rev. Lett. 1991, 67, 85.

(13) Hajduk, D. A.; Harper, P. E.; Gruner, S. M.; Honecker, C. C.; Kim, G.; Thomas, E. L.; Fetters, L. J. Macromolecules 1994, 27, 4063 .

(14) Schulz, M. F.; Bates, F. S.; Almdal, K.; Mortensen, K. Phys. Rev. Lett. 1994, 73, 86.

(15) Luzzati, V.; Tardieu, A.; Gulik-Krzywicki, T.; Rivas, E.; ReissHusson, F. Nature 1968, 220, 488. Luzzati, V.; Mariani, P.; Gulik-Krzywicki, T. In Physics of Amphiphilic Layers; Meunier, J., Langevin, D., Boccara, N., Eds.; Springer-Verlag: Berlin, 1987.

(16) Kajiyama, M.; Kakimoto, M.; Imai, Y. Macromolecules 1989, 22,$4143 ; 1990,23,1244$.

(17) Jho, J. Y.; Yee, A. F. Macromolecules 1991, 24, 1590.

(18) Benoit, H.; Hadziioannou, G. Macromolecules 1988, 21, 1449.

(19) Kavassalis, T. A.; Whitmore, M. D. Macromolecules 1991, 24, 5340.

(20) Zielinski, J. M.; Spontak, R. J. Macromolecules 1992, 25, 653.

(21) Matsen, M. W.; Schick, M. Macromolecules 1994, 27, 187.

(22) Helfand, E.; Wasserman, Z. R. In J. Developments in Block Copolymers -1; Goodman, I., Ed.; Applied Science: London, 1982.

(23) Gehlsen, M. D.; Almdal, K.; Bates, F. S. Macromolecules 1992, 25, 939. Hadziioannou, G.; Skoulios, A. Macromolecules 1982, $15,258$.

(24) Shull, K. R. Macromolecules 1992, 25, 2122.

(25) Shull, K. R.; Mayes, A. M.; Russell, T. P. Macromolecules 1993, $26,3929$.

(26) Semenov, A. N. Sov. Phys. JETP 1985, 61, 733.

(27) Vavasour, J. D.; Whitmore, M. D. Macromolecules 1992, 25, 5477.

(28) Anderson, D. M.; Thomas, E. L. Macromolecules 1988, $21,3221$.

(29) Spontak, R. J.; Zielinski, J. M. Macromolecules 1993, 26, 396.

(30) Semenov, A. N. Macromolecules 1993, 26,6617; see ref 21 within.

(31) Spontak, R. J.;Zielinski, J. M.; Lipscomb, G. G. Macromolecules $1992,25,6270$.

(32) Hong, K. M.; Noolandi, J. Macromolecules 1981, 14, 727.

(33) Leibler, L. Macromolecules 1980, 13, 1602.

(34) Helfand, E. J. Chem. Phys. 1975, 62, 999.

(35) The unnormalized basis functions for various structures can be found in: International Tables for X-Ray Crystallography; Henry, N. F. M., Lonsdale, K., Eds.; Kynoch: Birmingham, U.K., 1969. Those for the OBDD and G phases appear on pp 515 and $524-525$, respectively.

(36) Bates, F. S.; Schulz, M. F.; Rosedale, J. H.; Almdal, K. Macromolecules 1992, 25, 5547.

(37) Bates, F. S. Science 1991, 251, 898.

(38) Helfand, E.; Tagami, Y. J. Chem. Phys. 1972, 56, 3592.

(39) When comparing the multiblock system to the diblock system, we use $N / 2$ for the degree of polymerization of the diblocks.

(40) Matsen, M. W.; Schick, M. Phys. Rev. Lett. 1994, 72, 2660; Macromolecules 1994, 27, 4014 . 